\title{
INSTITUCIONES EDUCATIVAS UNIDAS POR UNA EDUCACIÓN MÁS EMOCIONAL
}

\author{
Esther Edo Agustín \\ Facultad de Ciencias Sociales y Humanas de Teruel \\ Universidad de Zaragoza \\ eedoa@unizar.es \\ Virginia Domingo Cebrián \\ Facultad de Ciencias Sociales y Humanas de Teruel \\ Universidad de Zaragoza
}

Recepción Artículo: 22 enero 2020

Admisión Evaluación: 4 marzo 2020

Informe Evaluador 1: 1 abril2020

Informe Evaluador 2: 7 abril 2020

Aprobación Publicación: 20 abril 2020

\section{RESUMEN}

La investigación educativa de carácter exploratorio que se desarrolla consiste en la unión y colaboración entre diferentes instituciones de la provincia de Teruel: Facultad de Ciencias Sociales y Humanas, Administración Educativa y Centros Educativos de Infantil y Primaria. El proyecto en torno al cual se coordinan es el programa de Educación Responsable facilitado por el departamento de educación de la Fundación Botín. Se trata de una intervención educativa pionera en España cuyo eje vertebrador es la educación emocional. El objetivo principal es fomentar la inteligencia emocional del alumnado participante con el fin de asegurar su desarrollo integral.

Los recursos que el programa de Educación Responsable ofrece son formación de los profesionales implicados y recursos educativos para los centros participantes. Asimismo, la Facultad de Ciencias Sociales y Humanas, en colaboración con el Centro de Profesorado Ángel Sanz Briz y la Inspección Educativa, conforma un equipo de investigación, con profesores de las áreas de conocimiento de Psicología y Ciencias de la Educación, que se encarga de diseñar y llevar a cabo la formación de los docentes de los centros participantes y guiarles mientras implantan la experiencia educativa innovadora.

Además, la Facultad de Ciencias Sociales y Humanas, incorpora la educación emocional en algunas de las asignaturas de los planes de Magisterio de Educación Infantil y Primaria y en el Máster de Profesorado de Educación Secundaria; también oferta una línea de investigación para los Trabajos Fin de Grado y Fin de Máster que pretende poner en valor el potencial de una educación basada en el conocimiento, comprensión e impacto de las emociones.

La cooperación entre instituciones es esencial y este proyecto apuesta por educar con el corazón.

Palabras clave: educación emocional; innovación pedagógica; cooperación institucional; Teruel; magisterio 


\section{INSTITUCIONES EDUCATIVAS UNIDAS POR UNA EDUCACIÓN MÁS EMOCIONAL}

\section{ABSTRACT}

Educatioinal institutions united by a more emotional education. The educational investigation of exploratory nature consists of the cohesion and collaboration between different institutions in the province of Teruel: Faculty of Humanities and Social Sciences, Educational Administration and Schools of Infant and Primary Education. The programme around which they are coordinated is Education Responsible, provided by the department of education of Spain s Botin Foundation. It is an educational intervention, pioneer in Spain, whose central thrust is emotional education. The main aim is to foster emotional intelligence in the participant students in order to ensure their integral development.

The resources that Education Responsible programme offers are training of the participant professionals and educational resources for the schools. Likewise, Faculty of Humanities and Social Sciences in collaboration with the Teacher s Centre Ángel Sanz Briz and Educational Inspection, creates a research team, with the teachers of Psychology and Education, which is responsible for designing and providing the training in the schools and the teachers support while they are developing the innovative educational experience.

In addition, Faculty of Humanities and Social Sciences includes emotional education in some of the curricular subjects of Infant and Primary Degrees and Secondary Master Degree; it also offers a research line, for the Final Degrees and Master Degrees, which exploits the value of an education based on the knowledge, comprehension and impact of emotions.

The cooperation between institutions is essential and this project is a bid for education with heart.

Keywords: emotional education; pedagogical innovation; institutional collaboration; Teruel; teaching

\section{INTELIGENCIA EMOCIONAL Y EDUCACIÓN EMOCIONAL}

La diversidad de enfoques desde los que se ha estudiado la influencia de las emociones en el desarrollo personal ha provocado la emergencia de múltiples estudios científicos a lo largo de las últimas décadas. A mediados de los años noventa comienzan a construirse nuevas conceptualizaciones del término inteligencia, que introducen en las variables a analizar las emociones y su influencia en el desarrollo cognitivo, físico, social e intelectual de Ios individuos (Binet y Simon,1961; Bar-on 1997; Marina, 1999). El término de inteligencia emocional es acuñado por Salovey y Mayer (1990) y definido como la capacidad de identificar, comprender y expresar las emociones, incluyen también en su definición la regulación de las mismas; los componentes que ambos introducen en su modelo de inteligencia emocional engloban desde habilidades de percepción e identificación emocional, hasta aquellas relacionadas con la gestión y regulación emocional. Asimismo, el modelo de inteligencia emocional desarrollado por Goleman (1996), a lo largo de la última década de los noventa, ejerce también una influencia fundamental en la extensión y popularización del término. Goleman (1999) establece la distinción entre competencias personales y sociales, enfatizando así el rol de las habilidades sociales y la empatía, elementos fundamentales para poder desenvolverse eficazmente diferentes contextos sociales.

En este sentido es posible afirmar que la adquisición de competencias emocionales es un proceso continuo que requiere experiencia para aprender a reconocerlas y experimentar con ellas, su desarrollo y control implica, por tanto, un proceso que se extiende a lo largo de la vida. La importancia de este proceso de adquisición tiene conexión directa no solo con la satisfacción y confort personal, sino también con la calidad de las interacciones (Brackett, Rivers, Shiffman, Lerner y Salovey, 2006). Destacan también múltiples estudios que establecen relaciones directas entre ser competente emocionalmente y alcanzar mayor éxito social y profesional; algunos de estos estudios demuestran que individuos con buenas competencias emocionales son capaces de solventar conflictos sociales de manera más eficaz (Zeidner y Kloda, 2013). Estudios similares identifican comportamientos menos agresivos en individuos con mayor inteligencia emocional (García-Sancho, Salguero y Fernández-Berrocal, 2014) y también mayores habilidades de liderazgo (Muñoz de Morales y Bisquerra, 2007).

Como consecuencia de los estudios publicados sobre inteligencia emocional, el valor de la educación emocional en los sistemas educativos de la actualidad se ha incrementado notablemente. Los profesionales de la educación reconocen el potencial de su desarrollo desde edades tempranas, razón por la cual, se concibe como pilar 
fundamental en los currículums educativos y se afirma que la escolarización continua y permanente debe promover el desarrollo de las competencias emocionales en los discentes, con el fin de mejorar su bienestar personal y capacitarles para la convivencia en sociedad (Bisquerra, 2010).

La actual tendencia educativa plantea la necesidad de concebir la educación emocional no solo como un trabajo transversal a desempeñar por los docentes, sino como un proceso educativo que implique al conjunto de la comunidad educativa; profesorado, familias y alumnado trabajando de manera cooperativa por la consecución de una sociedad más inteligente emocionalmente (Pinos, 2019). Para lograr de este objetivo común, no se debe obviar la importancia de la colaboración entre instituciones; Administración educativa, centros escolares y universitarios deben partir del mismo enfoque, que el alumnado pueda desarrollar sus competencias emocionales en entornos seguros y enriquecedores; para ello, es fundamental que el profesorado esté sensibilizado con la educación emocional y posea formación y recursos adecuados para poder desarrollar las competencias emocionales en su alumnado (Alcalá, Pérez et al., 2018)

\section{PROGRAMA DE EDUCACIÓN RESPONSABLE DE LA FUNDACIÓN BOTÍN}

Como hemos visto en el apartado previo, numerosos estudios, en diferentes contextos, aportan resultados que indican que trabajar las emociones de una forma sistemática ayuda a que las personas resuelvan mejor los problemas interpersonales, sean menos agresivas y, en general, tengan mejores indicadores de salud y bienestar psicológico y social.

Con esta filosofía, la Fundación Botín ha desarrollado un programa educativo cuyo objetivo es favorecer el crecimiento físico, emocional, social y creativo del alumnado, promover la comunicación y mejorar la convivencia en los centros escolares implicando adolescentes, alumnado y familias. El programa recibe el nombre de Educación Responsable (ER) y está implantado en más de 185 centros educativos de España y Latinoamérica.

El programa está compuesto por tres bloques: desarrollo emocional, desarrollo cognitivo y desarrollo social. Todos ellos se ejecutan teniendo muy presente la creatividad. (Véase figura 1)

Figura 1. Bloques del contenido del programa ER

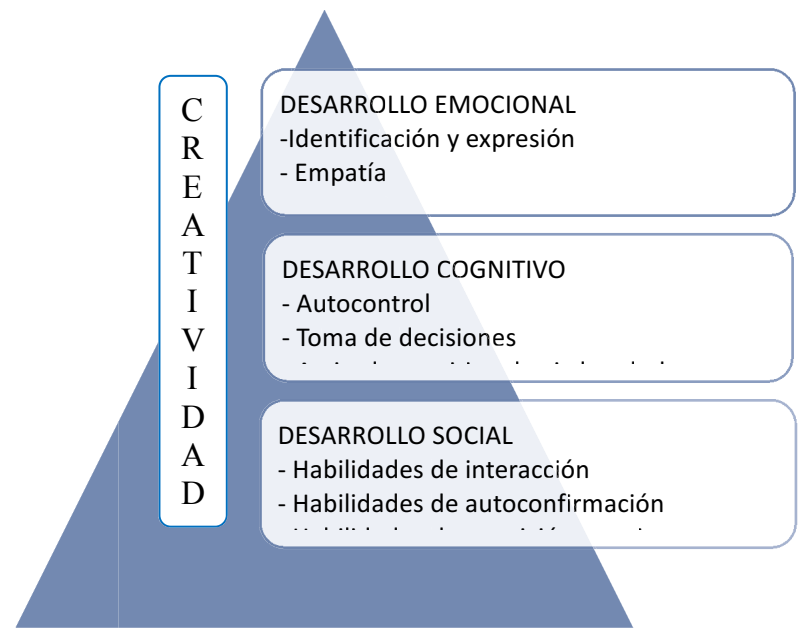




\section{INSTITUCIONES EDUCATIVAS UNIDAS POR UNA EDUCACIÓN MÁS EMOCIONAL}

Estos tres bloques se planifican para implementarlos a lo largo de tres cursos académicos (véase tabla 1). Además, para que esto pueda realizarse con garantías es necesario tener recursos y una buena formación. Aquí es donde interviene el equipo base, que está formado por profesorado universitario, concretamente de la FCSH, y pertenecen a las áreas de conocimiento de Psicología y Ciencias de la Educación. Ellos apoyan, guían y orientan a los maestros de los centros educativos participantes.

Tabla 1

Fases del programa de ER

\begin{tabular}{|l||l||l||}
\hline \multicolumn{1}{|c|}{$\begin{array}{c}\text { AÑO 1. (2018/2019) } \\
\text { FASE INICIAL }\end{array}$} & $\begin{array}{c}\text { AÑO 2. (2019/2020) } \\
\text { FASE DE EXPANSIÓN }\end{array}$ & $\begin{array}{l}\text { AÑO 3. (2020/2021) } \\
\text { CONSOLIDACIÓN }\end{array}$ \\
\hline $\begin{array}{l}\text { Formación presencial del } \\
\text { profesorado: Módulo } \\
\text { Desarrollo Afectivo. }\end{array}$ & $\begin{array}{l}\text { Creación de grupos de } \\
\text { trabajo. }\end{array}$ & $\begin{array}{l}\text { Extensión y generalización } \\
\text { de grupos de trabajo. }\end{array}$ \\
\hline \begin{tabular}{l|l|l} 
Formación a través de los \\
recursos del campus \\
virtual de Educación \\
Responsable.
\end{tabular} & $\begin{array}{l}\text { Formación presencial del } \\
\text { profesorado: Módulo } \\
\text { Desarrollo Social. }\end{array}$ & $\begin{array}{l}\text { Formación presencial del } \\
\text { profesorado: Módulo } \\
\text { Desarrollo Creatividad. }\end{array}$ \\
\hline
\end{tabular}

En los centros educativos, el programa ER, queda planificado dentro de sus programaciones didácticas. Concretamente se involucran a la tutoría y a algunas áreas curriculares. Se aplica desde Educación Infantil hasta la Educación Secundaria Obligatoria (ESO). Asimismo, se extiende también al ámbito familiar a través de algunas actividades complementarias.

Los recursos que ofrece el programa de ER (véase tabla 2) son los siguientes:

- Banco de Herramientas (BdH), da la oportunidad de trabajar la educación emocional en el aula a partir de tres elementos: propuestas para la promoción del bienestar y el desarrollo integral del alumnado, soportes audiovisuales y técnicas de trabajo en grupo. Generalmente, las actividades que se plantean se desarrollan en horario de tutoría para las etapas de Primaria y ESO, en Infantil se trabaja de forma globalizada.

- Lectura y emociones, es un recurso para acompañar a los alumnos de Educación Infantil en su educación emocional a través de cuentos. Se plantea de manera globalizada, pues abarca las tres áreas de conocimiento del currículo.

- Literatura, emociones y creatividad, es un recurso que, a través de libros, trabaja el desarrollo emocional, cognitivo y social, y la creatividad. En las etapas de Primaria y ESO, se desarrolla en el área de Lengua castellana y literatura.

- El coro de las emociones, utiliza el canto coral como herramienta lúdica y al alcance de cualquiera para trabajar contenidos musicales y contribuir al desarrollo emocional, social y de la creatividad. Se plantea globalizada en infantil, en Educación Primaria se realiza en el área de Educación artística y en ESO en el área de Música.

- ReflejArte, es un recurso educativo que, a través de las artes plásticas, trabaja la educación emocional, social y la creatividad. El trabajo se divide en tres fases interrelacionadas y consecutivas, articuladas en torno a un autor y obra. En Educación Primaria se realiza en el área de Educación artística y en ESO en el área de Educación plástica, visual y audiovisual. 
Tabla 2

Programación de los recursos

\begin{tabular}{|l|l|l|}
\hline \multicolumn{1}{|c|}{ RECURSOS } & ETAPAS EDUCATIVAS & TEMPORALIZACIÓN \\
\hline BdH & Infantil, Primaria y ESO & Todo el curso \\
\hline Lectura y emociones & Infantil & Segundo trimestre \\
\hline $\begin{array}{l}\text { Literatura, emociones y } \\
\text { creatividad }\end{array}$ & Primaria y ESO & Segundo trimestre \\
\hline El coro de las emociones & Infantil, Primaria y ESO & Tercer trimestre \\
\hline ReflejArte & Primaria y ESO & Primer trimestre \\
\hline
\end{tabular}

Con la aplicación del programa de ER se pretende conseguir los siguientes resultados:

- Que el alumnado mejore la identificación y comprensión de sus emociones, así como su creatividad.

- Que el profesorado observe en el alumnado una disminución de comportamientos agresivos y de retraimiento social, así como disminución de la ansiedad.

- Que los centros educativos experimenten una mejora de la convivencia, el clima escolar y el bienestar del alumnado.

- Que las familias perciban una mejora en valores y conductas prosociales como la generosidad, la empatía y la colaboración.

\section{¿EXISTE FORMACIÓN SOBRE EDUCACIÓN EMOCIONAL EN LOS PLANES DE ESTUDIO DE MAGIS- TERIO?}

La formación inicial docente y permanente viene regulada en España por la Ley Orgánica 2/2006, de 3 de mayo, de Educación en su CAPÍTULO III sobre la Formación del profesorado, en los artículos 100 y 102. El proceso formativo del maestro está condicionado por las influencias de la política educativa, por lo que se considera a un maestro competente según el desarrollo profesional.

Actualmente, el modelo pedagógico vinculado a las enseñanzas universitarias ha pasado de ser un modelo basado en la docencia a un sistema basado en el aprendizaje por competencias. En los programas de enseñanza-aprendizaje, el alumnado es el eje principal y las asignaturas y módulos planteados tienen que estar ligados al progreso de sus capacidades.

Asimismo, no solo hay que tener presentes las competencias en la formación inicial docente, sino que hay que reflexionar sobre el perfil del estudiante que llega a los estudios de Magisterio, su nivel de motivación, actitud, aptitud y autoeficacia. El informe TALIS 2018 indica que una problemática que presentan muchos países es la dificultad para atraer a la profesión docente a individuos que estén altamente cualificados y motivados a la vez. En relación a esto, las investigaciones sugieren que hay ciertos aspectos en los programas de preparación de nuevos docentes que pueden marcar la diferencia, como son la duración, el contenido y la certificación de estos programas. (Darling- Hammond, 2000).

Según Carbonero et al (2009), resulta clave atender a los cambios sociales que demandan habilidades docentes motivadoras para los futuros profesionales y también la inclusión de una interpretación más holística que incluya el enfoque teórico-práctico incentivado de dimensión personal (Korthagen, 2010).

Algunos autores (Bisquerra, 2007; López-Goñi y Goñi, 2012) califican de acertada la inclusión de las competencias emocionales en la formación inicial del docente, aunque en la práctica ocupan un segundo o tercer plano dentro del conjunto de las competencias docentes. En este sentido, Gallego (2004) señalan que la adecuada gestión emocional y la armonización entre lo cognitivo y lo emocional debe contemplarse plenamente en el currículum. Además, Livia y García (2015) exponen cómo el contexto educativo representa un lugar privilegiado para la promoción de las habilidades y el desarrollo de competencias socioemocionales, las cuáles contribuyen de forma positiva al bienestar personal y social de las personas. 


\section{INSTITUCIONES EDUCATIVAS UNIDAS POR UNA EDUCACIÓN MÁS EMOCIONAL}

Para Bisquerra (2007), el profesorado emocionalmente competente está mejor preparado para relacionarse de manera positiva y adecuada con la comunidad educativa, aumentando la eficiencia de la educación.

En consonancia con los autores y dando respuesta a la pregunta planteada al inicio de este apartado, la FCSH, ha incorporado la educación emocional en algunas asignaturas de los planes de estudio de Magisterio de Educación Infantil y Primaria. Además, se ha ofertado una línea de investigación de dicha temática para la realización de Trabajos Fin de Grado (TFG) y de Trabajos Fin de Máster (TFM), este último para los estudiantes del Máster de Profesorado de Educación Secundaria Obligatoria, Bachillerato, Formación Profesional y Enseñanzas de Idiomas, Artísticas y Deportivas. Todo esto ha sido posible, por un lado, por la participación del profesorado universitario en el programa de ER, y por otro, porque las guías docentes de las asignaturas seleccionadas permiten a través de sus competencias vincularlas estrechamente a la temática emocional.

En concreto, las asignaturas seleccionadas han sido:

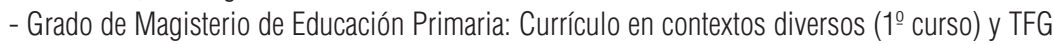

- Grado de Magisterio de Educación Infantil: El maestro en la escuela infantil $\left(2^{0}\right.$ curso) y TFG

- Máster de Profesorado de Educación Secundaria Obligatoria, Bachillerato, Formación Profesional y Enseñanzas de Idiomas, Artísticas y Deportivas: TFM.

\section{OBJETIVOS}

Aplicar e integrar el programa de ER en los centros piloto, para promover el desarrollo saludable de las personas y mejorar la convivencia y la comunicación en los centros a partir del trabajo con docentes, alumnado y familias.

Adquirir recursos para capacitar al profesorado a trabajar con su alumnado la Inteligencia Emocional y el desarrollo de competencias personales como identificación y expresión emocional, empatía y autoestima.

Introducir la educación emocional en la formación inicial docente.

Establecer una línea de investigación para que alumnado de magisterio y de máster de profesorado pueda desarrollar sus TFG y TFM con temáticas relacionadas con la educación emocional.

\section{Participantes}

Los sujetos que forman parte de esta experiencia educativa innovadora son tanto alumnado de la FCSH (Grado de Magisterio en Educación Infantil, Grado de Magisterio en Educación Primaria y Máster Universitario en Profesorado de ESO, Bachillerato, F.P. y Enseñanzas de Idiomas, Artísticas y Deportivas), como profesorado de Centros de Educación Infantil y Primaria, Colegio Rural Agrupado y Centro Rural de Innovación Educativa. También participan activamente profesores universitarios del departamento de Ciencias de la Educación y departamento de Psicología y Sociología de la FCSH, Asesores del Centro de Profesores Ángel Sanz Briz e inspectores del Servicio Provincial de Educación de Teruel. Conviene destacar que todos los profesionales de la educación implicados en la experiencia, participan voluntariamente en la misma, con el incentivo de conseguir de la educación emocional, una realidad educativa en los centros de la provincia de Teruel. 
Figura 1. Número de participantes por instituciones.

\begin{tabular}{|c|c|c|c|c|c|c|c|c|c|}
\hline 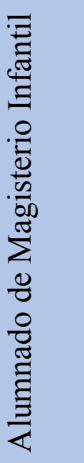 & 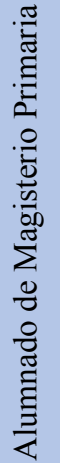 & 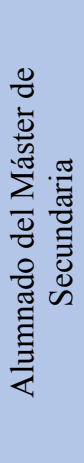 & 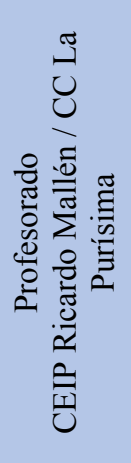 & 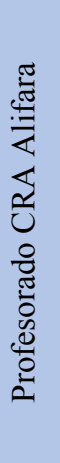 & 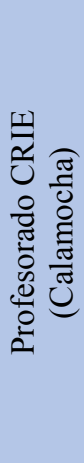 & 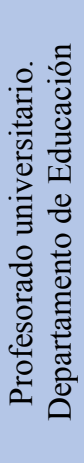 & 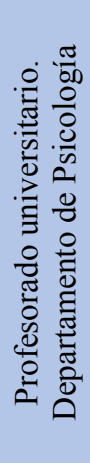 & 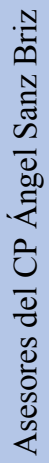 & 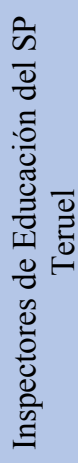 \\
\hline 152 & 121 & 2 & 55 & 20 & 6 & 4 & 3 & 1 & 2 \\
\hline
\end{tabular}

\section{Metodología e instrumentos}

La metodología utilizada es de corte mixto. Por un lado, tiene carácter cuantitativo a través de la técnica de encuesta con modelo escala Likert que se aplica a los docentes una vez han finalizado los periodos de formación. Por otro lado, es cualitativa al utilizarse espacios dialógicos o de reflexión (Brockbank \& McGill, 2006; Freire, 1997), con estos mismos docentes para valorar la formación a través de los recursos del campus virtual de ER y con los estudiantes de los Grados de Magisterio de Educación Infantil y Primaria.

Los espacios de reflexión entre el profesorado participante han sido múltiples y variados. Cabe destacar la formación inicial impartida por la Fundación Botín durante el mes de septiembre tanto del curso 2018/2019 como del 2019/2020. En las sesiones impartidas se trabajan contenidos teóricos relacionados con las variables que el Programa de ER engloba. Concretamente las sesiones de formación inicial impartidas a comienzo del curso 2018/2019 para los equipos base se centraron en el Módulo I. Desarrollo Afectivo; mientras que las sesiones de formación del equipo base del curso 2019/2020 desarrollaron el Módulo II. Desarrollo Social. Tras dichas sesiones iniciales, los equipos base se reúnen y acuerdan los contenidos a desarrollar y en colaboración con el asesor del Centro de Profesorado establecen las fechas de las formaciones en los centros.

Las formaciones impartidas por los equipos base son evaluadas por el profesorado de los centros educativos participantes a través de encuestas Google Form, las cuales cuentan con preguntas cerradas y abiertas. Las primeras comprenden variables como interés por los contenidos, calidad docente, satisfacción global, sesiones prácticas, metodología empleada y cumplimiento de expectativas, la escala de respuesta numérica tipo Likert es de 1 a 5 puntos ( 1 = muy bajo / 5 = muy alto). Las segundas valoran aspectos como utilidad de la formación recibida por el equipo base, elementos positivos y propuestas de mejora.

Además, los coordinadores del Programa de ER de la Provincia de Teruel, aprovechan también el inicio de curso para reunirse con el profesorado participante de los centros escolares, en dichas sesiones se establecen nuevamente sinergias con el objeto de fomentar la educación emocional y tratar de implicar a toda la comunidad educativa en este proceso. A final del curso escolar, los coordinadores vuelven a convocar al profesorado participante y en colaboración con los equipos base y tutores del campus virtual, intercambian reflexiones sobre los recursos, analizan sus ventajas / limitaciones y sugieren propuestas de mejora.

Asimismo, con los estudiantes de los Grados de Magisterio de Educación Infantil y Primaria, se han Ilevado a cabo espacios de reflexión dentro de las asignaturas de Currículo en contextos diversos y El maestro en la escuela infantil. En la planificación de las asignaturas el profesorado ha incluido la educación emocional y a tra- 


\section{INSTITUCIONES EDUCATIVAS UNIDAS POR UNA EDUCACIÓN MÁS EMOCIONAL}

vés de una serie de tareas los estudiantes han podido conocer en qué consiste. Posteriormente, en las puestas en común se abrían estos espacios de diálogo y/o reflexión.

\section{Resultados}

Los resultados de los espacios dialógicos y/ de reflexión entre los estudiantes han sido un éxito. Ellos han sido los verdaderos protagonistas de su aprendizaje, utilizando metodologías más activas e innovadoras y dando la importancia que se merece a la reflexión; valorando así la gran transcendencia que tiene su papel en la sociedad, formando a los ciudadanos del futuro. Además, les ha permitido fomentar el deep learning, que permite la transferencia de los conocimientos entre asignaturas y de la teoría a la práctica, así como adquirir hábitos de pensamiento, de las emociones y de la acción (Marina, Pellicer, Manso, 2015). Asimismo, en el curso 2018/2019 se defendieron tres TFG afines a la temática y en el presente curso están en proceso dos TFG y dos TFM.

Los resultados de los espacios de reflexión entre profesionales de la educación indican un grado positivodestacado de satisfacción del profesorado hacia la formación recibida por los equipos base. El carácter innovador de la experiencia y la relevancia de los contenidos impartidos provocan un incremento en la motivación del profesorado participante, tanto de los centros escolares, como del profesorado universitario que recibe e imparte la formación. Además, conviene también indicar, atendiendo a las propuestas de mejora del profesorado de los centros escolares, que las formaciones recibidas podrían aportar un enfoque más práctico y transferible a las realidades educativas de los centros participantes, algunos de ellos emplazados en contextos rurales.

Los recursos del campus virtual son valorados también positivamente por el profesorado de los centros escolares, destacan principalmente la amplia diversidad de actividades facilitadas a través del BdH y clasificadas en diferentes niveles educativos. Entre las propuestas de mejora que emergieron durante la puesta en común de la experiencia al finalizar el curso escolar 2018/2019, se puede destacar la creación de nuevos espacios y tiempos de interacción entre los centros escolares participantes.

\section{DISCUSIÓN Y CONCLUSIONES}

La posibilidad de trabajar conjuntamente y de manera coordinada entre diferentes instituciones relacionadas con el ámbito educativo impacta positivamente sobre la comunidad educativa. Una experiencia como la expuesta, en la que participan centros educativos, Administración Educativa y Universidad, adquiere un alto valor en la educación actual.

Queda constatada la satisfacción de los participantes, quienes indican, en líneas generales, que la experiencia les ha permitido crecer a nivel personal y profesional, gracias a la formación en educación emocional, viendo mejorado a su vez el clima escolar de los centros.

Asimismo, el alumnado de magisterio de la FCSH, también ha valorado positivamente su inmersión en dicha temática; puesto que les ha permitido adquirir nuevos recursos, reflexionar sobre la practica educativa actual y tomar conciencia de la relevancia de su profesión en la sociedad. Tanto es así, que se ha visto incrementado el interés por realizar TFG y TFM en líneas de investigación de educación emocional.

No debemos olvidar que las emociones forman parte de nuestras vidas, por lo tanto, también deben formar parte de nuestra educación.

\section{Agradecimientos}

Los datos presentados en este estudio tienen financiación de la Universidad de Zaragoza a través de la convocatoria de Proyectos de Innovación Docente para Grupos de Profesores (PIIDUZ_19_257).

\section{REFERENCIAS BIBLIOGRÁFICAS}

Alcalá, M.L, Pérez, Y. et al (2018). Enseñando con lápiz y corazón: proyecto de educación emocional en la etapa de Educación Infantil, Teruel. Sitio web Educaragón: http://www.educaragon.org/FILES/ Enseñando\%20con\%20Lápiz\%20y\%20Corazón.pdf 
Bar-On, R. (1997). The emotional quotient inventory: Technical manual. MultiHealth Systems, Inc.

Binet, A., \& Simon, T. (1961). The Development of Intelligence in Children. In J. J.

Jenkins \& D. G. Paterson (Eds.), Studies in individual differences: The search for intelligence, 81-111.

Bisquerra, R. (2007). La educación emocional en la formación del profesorado. Revista Interuniversitaria de Formación del Profesorado, 54, 95-114.

Bisquerra, R. (2010). La educación emocional en la práctica. Horsori Editorial S.L.

Brackett, M. A., Rivers, S. E., Shiffman, S., Lerner, N. \& Salovey, P. (2006). Relating emotional abilities to social functioning: A comparison of self-report and performance measures of emotional intelligence. Journal of Personality and Social Psychology, 91(4), 780-795.

Brockbank, A. \& McGill, I. (2006). Facilitating reflective learning through mentoring and coaching. Kogan Page.

Carbonero, M. A., Román, J. Mª., Martín-Antón, L. J., Reoyo, N. (2009). Efecto del programa de habilidades docentes motivadoras en el profesorado de secundaria Revista de Psicodidáctica, 14 (2), 229-243.

Darling-Hammond, L. (2000). Teacher Quality and Student Achievement: A Review of State Policy Evidence. Education Policy Analysis Archives, 8 (1), 1-44.

Freire, P. (1997). Pedagogía de la autonomía. Siglo XX

Gallego, D.J. y Gallego, M.J. (2004). Educar la inteligencia emocional en el aula. PPC

García-Sancho, E., Salguero, J. M., \& Fernández-Berrocal, P. (2014). Relationship between emotional intelligence and aggression: a systematic review. Aggress. Violent

Behav, 19, 584-591.

Goleman, D. (1996). La inteligencia emocional. Kairós.

Goleman, D. (1999). La práctica de la inteligencia emocional. Kairós.

Korthagen, A.J. (2010). La práctica, la teoría y la persona en la formación del profesorado. Revista Interuniversitaria de Formación del Profesorado, 24(2), 83-101

Livia, G. y García, L. (2015). Estudio de las competencias socio-emocionales y su relación con el afrontamiento en futuros profesores de nivel medio. REIFOP. Revista Electrónica Interuniversitaria de Formación del Profesorado, 18(1), 213-228.

López-Goñi, I. y Goñi, J.M. (2012). La competencia emocional en los currículos de formación inicial de los docentes. Un estudio comparativo. Revista de Educación, 357, 205-206.

Marina, J.A., Pellicer, C. y Manso, J. (2015). Libro blanco de la profesión docente y su entorno escolar. UAM

Marina, J. A., y Penas, M. L. (1999). Diccionario de los sentimientos. Anagrama.

Ministerio de Educación y Formación Profesional (2018). TALIS. Estudio internacional de la enseñanza y del aprendizaje. Informe español. Madrid. Sitio web MEFP: https://www.educacionyfp.gob.es/dam /jcr:047dfc41-acf6-444a-8e4e-4e5916343a88/talis2018-online-20190807.pdf

Muñoz de Morales, M., y Bisquerra, R. (2013). Diseño, aplicación y evaluación de un plan de educación emocional en Guipúzcoa: análisis cuantitativo. EduPsykhé,12, 3-21.

Pinos, M. (2019). Con corazón y cerebro: Net learning: aprendizaje basado en la neurociencia, la emoción y el pensamiento. Caligrama.

Salovey, P., \& Mayer, J. D. (1990). Emotional intelligence. Imagination, cognition and personality, 9(3), 185211.

Zeidner, M., \& Kloda, I. (2013). Emotional intelligence (El), conflict resolution patterns, and relationship satisfaction: actor and partner effects revisited. Pers. Individ. Differ, 54, 278-283. 
